\title{
Research on Conflict Decision between Shift Schedule and Multienergy Management for PHEV with Automatic Mechanical Transmission under Special Driving Cycles
}

\author{
JunQiang $X i^{1}$ and Yongdan Chen ${ }^{2}$ \\ ${ }^{1}$ School of Mechanical Engineering, Beijing Institute of Technology, Beijing 100081, China \\ ${ }^{2}$ China North Vehicle Research Institute, Beijing 100072, China \\ Correspondence should be addressed to JunQiang Xi; xijunqiang@bit.edu.cn
}

Received 30 September 2013; Accepted 3 November 2013

Academic Editor: Hui Zhang

Copyright (C) $2013 \mathrm{~J}$. Xi and Y. Chen. This is an open access article distributed under the Creative Commons Attribution License, which permits unrestricted use, distribution, and reproduction in any medium, provided the original work is properly cited.

In order to satisfy the character of parallel hybrid electric vehicle (PHEV) in some special driving cycles, a collision decision problem between the shift decision and power split ratio is proposed. Based on a large amount of experimental data the optimal decisions are determined with evidential reasoning theory. The proposed decision strategy has been verified through real road test of Chongqing public transportation line 818 and the fuel economic improvement has also been achieved.

\section{Introduction}

The so-called special driving cycles of hybrid buses traveling refers primarily to climbing a big slope and/or rapid acceleration (or kickdown). A common feature in these cycles is the requirement of large drive torque to overcome road resistance or to provide additional acceleration. This is similar to the Boost function applied in some passenger cars. To provide short-term high torque in the traditional vehicles, in addition to extracting engine power, an increased gear ratio is used. But the engine cannot be overloaded due to existing selfsustaining measures; hence the torque response is slow. Thus in order to meet the requirement of large torque in a short time, traditional vehicles commonly use the method of power downshifting.

When parallel hybrid electric bus is faced with such special driving cycles, the traction motor of hybrid powertrain allows short-term overload. In this case there are two mutually exclusive decision-making options: (1) multienergy power spilt management and (2) gear changing. This leads to a task conflict, since both the decision-making schedules involve the motor control that is not feasible to realize simultaneously. Although the regulation of the Chinese urban road design code CJJ37-1990 states that "the maximum longitudinal limiting value is $9 \%$ in the urban road [1]," in some mountain cities (such as Chongqing etc.) it is $15 \%$. This can be attributed to the influence of topographical factors, which implies that the bus often needs to climb a big slope and the up gradient will cause a deceleration of nearly $1.5 \mathrm{~m} / \mathrm{s}^{2}$.

If the shift operation occurs during this gradient climb and the time of torque interruption is 2 seconds, the speed will decrease by $10.8 \mathrm{~km} / \mathrm{h}$. This decrease in speed is difficult to be accepted in terms of ride comfort running performance. In addition, if the downshift operation is selected during the undulating road, there is a chance that automatic transmission might start the upshift operation as soon as a certain speed level is reached. This can bring forth the phenomenon of shift hunting (undesirable). Conversely, if multienergy power spilt management is selected to increase the motor drive torque in such a case then the shift hunting phenomenon can be avoided. Further study about the problems is required such as (1) whether the use of electrical energy is reasonable and (2) whether the maximization of the vehicle performance could be realized.

At present, many scholars have argued that the key to solve such problems is pattern recognition, so in-depth and 
meticulous research has been conducted on pattern recognition $[2,3]$. In the case of hybrid vehicles, even if obtaining information of the external pattern is available, the problem of making the optimal decision exists. Therefore, there is an urgent need to research on shift schedule and multi-energy power split management decision-making under special driving cycles for hybrid buses. This paper will study the problem of task conflict in hybrid buses while climbing big slope by applying the multiattribute decision-making theory.

\section{Mathematical Description of Task Conflict under Special Driving Cycles}

The decision-making process has to take into view the characteristics of hybrid buses' traveling road uncertainty, driving behavior randomness, and the dynamic nature of powertrain control. First of all, the problem of evaluation and decisionmaking must consider qualitative attributes. The so-called qualitative attribute judges the information of one vehicle properties, and the evaluation is not a specific value but a grade of linguistic assessment [4]. The collection of evaluation grades is given by

$$
H=\left\{H_{1}, \ldots, H_{n}, \ldots, H_{N}\right\},
$$

where $H_{n}$ assesses the grade of each attribute. To simplify the calculation, $H_{n}$ is quantized in a certain scale [5] and $p\left(H_{n}\right)$ stands for the grade of $H_{n}$. The evaluation grades' range is limited in $[-1,1]$ and will be quantized by

$$
p\{H\}=\left[p\left(H_{1}\right), \ldots, p\left(H_{n}\right), \ldots, p\left(H_{N}\right)\right]^{T} .
$$

A finite set of basic attributes are denoted by

$$
E=\left\{e_{1}, \ldots, e_{i}, \ldots, e_{L}\right\} .
$$

We assume that the weighting factor is

$$
W=\left(w_{1}, \ldots, w_{i}, \ldots, w_{L}\right), \quad 0 \leq w_{i} \leq 1,
$$

where $w_{i}$ is the weight suited for the respective basic attribute $e_{i}$, which plays a key role in multiple attribute decision-making. The evaluation of each attribute $e_{i}$ can be expressed as

$$
S\left(e_{i}\right)=\left\{\left(H_{n}, \beta_{n, i}\right) \mid n=1, \ldots, N\right\}, \quad \text { for } i=1, \ldots, L,
$$

where $\beta_{n, i}$ is the trust degree of correlation attribute and satisfies $\beta_{n, i} \geq 0, \sum_{n=1}^{N} \beta_{n, i} \leq 1$. When $\sum_{n=1}^{N} \beta_{n, i}=1, S\left(e_{i}\right)$ is called complete evaluation, when $\sum_{n=1}^{N} \beta_{n, i}<1, S\left(e_{i}\right)$ is called incomplete evaluation.

For convenience, assume that only the evaluation of twolevel attribute is considered, as is shown in Figure 1, define the vehicle performance $y$ as a total attribute of the upper, $E=$ $\left\{e_{1}, e_{2}, e_{3}, e_{4}, e_{5}, e_{6}, e_{7}, e_{8}, e_{9}\right\}$ as a basic attribute of the lower, and $\beta_{n}$ as the trust degree of evaluation grade for total attribute $y$. Specifically, the evaluation grade is divided into six classes; they are, respectively, as follows:

$$
\begin{aligned}
H=\{ & H_{1} \text { (worst) }, H_{2} \text { (poor) }, H_{3} \text { (average) }, \\
& \left.H_{4}(\text { Good }), H_{5} \text { (Excellent) }, H_{6}(\text { Top })\right\} .
\end{aligned}
$$

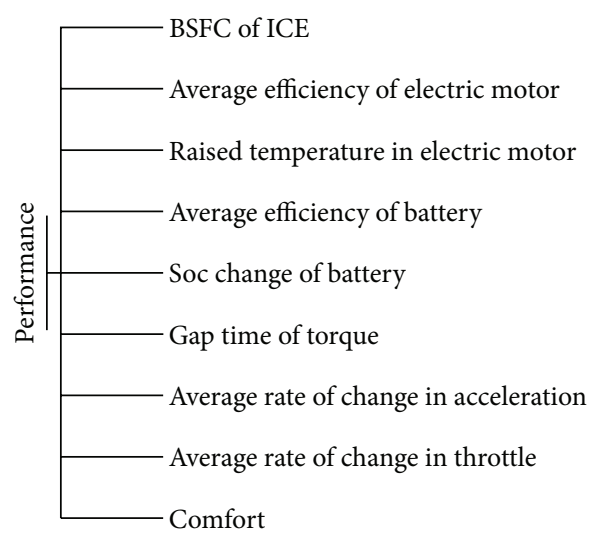

FIGURE 1: Evaluating hierarchy of Vehicular performance under special driving cycles.

According to the evaluation of expected vehicle performance of total attribute $y$ under various decision-making, the final decision of multi-energy power spilt management under special driving cycles is obtained.

\section{Data Acquisition of Basic Attribute for Hybrid Powertrain under Special Driving Cycles}

In order to evaluate the multi-attribute decision-making for hybrid electric bus under typical special driving cycles, it is needed to collect the impacts on vehicle performance when gear changing and multi-energy power spilt management are applied, respectively, under the typical driving cycle and different vehicle states. Taking climbing a big slope condition as an example to explain the tests designed to obtain the performance parameters of the same type of hybrid buses in test venue, the specific test process is shown in Figure 2. The specified value of each test in the flowchart is shown in Table 1 , and the recorded data is the basic attribute in Figure 1.

In order to ensure that the two strategies will be output of the same driving force on the vehicle, the motor output torque required in the test is decided by the difference between the output torque of low gear and current gear. The impact on vehicle performance after application of the shift schedule and multi-energy power split management strategy by the above-mentioned test under uphill condition is described by quantitative and qualitative data respectively.

Because of the uncertain factors which are brought about by the deviation of artificial control and the influence of external environment in the above-mentioned process, the test does not exactly match the actual operating conditions, and it causes uncertainty of the collected qualitative and quantitative data. A more effective method for this uncertainty is to synthesize the evidence (data) via Dempster-Shafer theory. The next section will introduce the Dempster-Shafer evidential reasoning approach. 
TABLE 1: Related values in test process.

\begin{tabular}{|c|c|c|c|c|c|}
\hline \multirow{2}{*}{$\begin{array}{l}\text { Category of specified value } \\
\text { Slope }(\%)\end{array}$} & \multicolumn{5}{|c|}{ Test value } \\
\hline & 5 & 10 & 13 & 16.7 & - \\
\hline $\operatorname{SOC}(\%)$ & 20 & 40 & 60 & 70 & 80 \\
\hline Vehicle speed passing slope bottom $(\mathrm{km} / \mathrm{h})$ & 10 & 15 & 20 & 30 & 50 \\
\hline Throttle opening (\%) & 40 & 50 & 60 & 80 & 100 \\
\hline
\end{tabular}

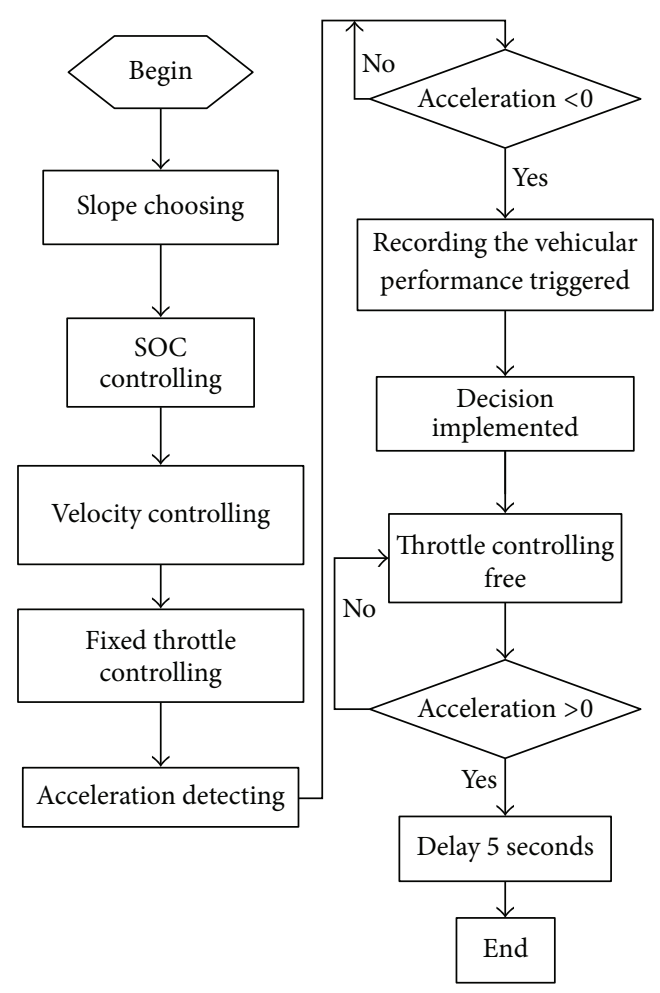

FIGURE 2: Multi-attribute data collecting flow under ramp condition.

\section{Conflict Task Decision-Making Based on the Evidential Reasoning Approach}

The evidential reasoning approach was first proposed by the University of Manchester, UK, Jian-Bo Yang in 1994; he first proposed a basic evidential reasoning model [6], which theoretical foundation is based on the Dempster-Shafer evidence theory as well as multi-attribute decision-making analysis framework [7].

\subsection{Basic Concept of Dempster-Shafer Evidence Combination} Theory. Evidence theory was first proposed by Dempster in 1967 and later was formally established on monograph "A Mathematical Theory of Evidence," which is published in 1976 by his student Shafer [8], known as the Dempster-Shafer evidence theory.

Define $\Theta=H$ as a frame of discernment; it is a collection of all possible results we can recognize about the hybrid bus performance, and the basic performance attributes of the vehicle are shown in Figure 1. The selection of them depends on our prior knowledge and experimental data, and the elements in the frame of discernment should be independent of each other [7]. The trust for proposition or decision-making based on some evidence is described by the mass function (basic probability assignment) [9]. The power set $2^{\Theta}$ in the frame of discernment $\Theta$ constitutes a collection of all possible vehicular states. Trust degree is given to all propositions (including $\Theta$ ) via mass function according to the obtained vehicular experimental data, which is the probability of separate appearance for similar vehicle state under different special driving cycles. Mass function is defined as the mapping of $m: 2^{\Theta} \rightarrow[0,1]$

$$
\begin{aligned}
& 2^{\Theta}=\left\{\phi,\left\{\Theta_{1}\right\}, \ldots,\left\{\Theta_{N}\right\},\left\{\Theta_{1}, \Theta_{2}\right\}, \ldots,\right. \\
&\left.\left\{\Theta_{N-1}, \Theta_{N}\right\}, \ldots,\left\{\Theta_{N-1}, \ldots, \Theta_{N}\right\}\right\}
\end{aligned}
$$

and satisfies the next two conditions:

$$
m(\phi)=0, \quad \sum_{A \in 2^{\Theta}} m(A)=1,
$$

where $\forall A \in \Theta, m(A)$ indicates the trust degree to proposition $A$; if $m(A)>0$, then $A$ is called focal element. For all of $A \neq \Theta$, if $m(\Theta)=1$ and $m(A)=0$, then it is called that $m$ has not been assigned in any subsets but is the expression for the unknown information.

For any proposition, it could obtain two evidential functions by the mass function, which, respectively, are the belief function Bel and the plausibility function $\mathrm{Pl}$ and are defined as follows [10]:

$$
\begin{gathered}
\operatorname{Bel}(A)=\sum_{\phi \neq B \subseteq A} m(B), \\
\operatorname{Pl}(A)=1-\operatorname{Bel}(-A)=1-\sum_{B \subseteq-A} m(B),
\end{gathered}
$$

where $\mathrm{Pl}(A)$ indicates the degree of reliability or plausibility for $A$ we can find. The sum of the trust function of a proposition and the trust function of its negative proposition does not necessarily equal to 1 ; that is,

$$
\operatorname{Bel}(A)+\operatorname{Bel}(-A) \leq 1 .
$$

The feature for D-S evidence theory is more excellent than the past fusion theory because it is established on its description of incident. It is not a single point probability value which is described with the traditional probability theory, but it possesses the trust interval $[\operatorname{Bel}(A), \operatorname{Pl}(A)]$. $\operatorname{Bel}(A)$ indicates the support degree of the evidence to this proposition; plausibility function $\operatorname{Pl}(A)$ indicates the degree by which the evidence does not deny this proposition. It 


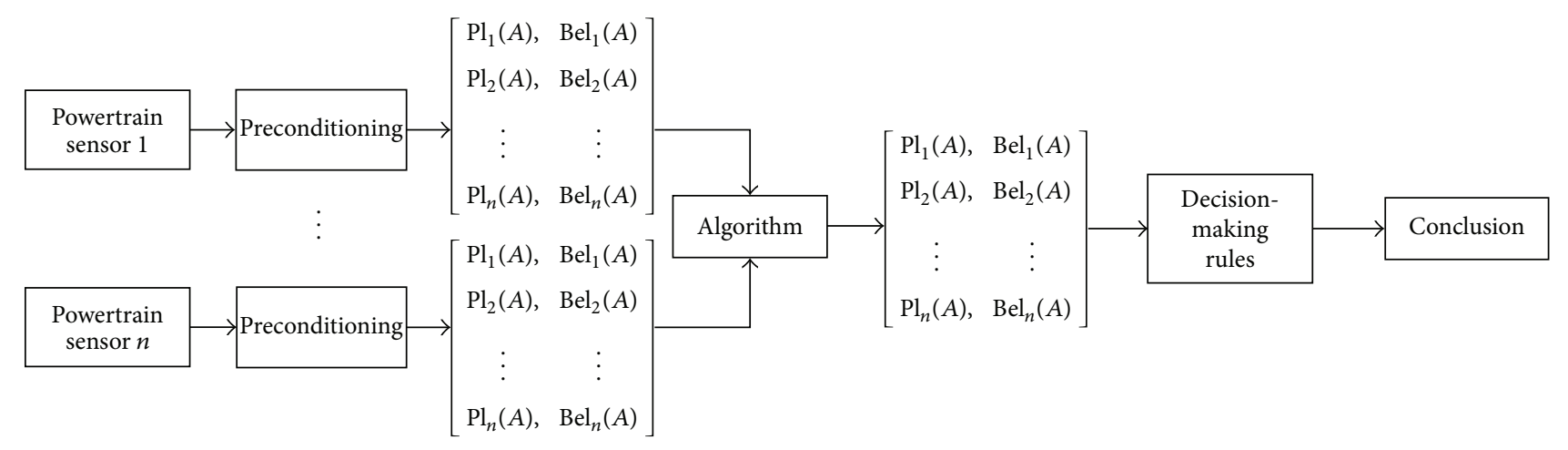

Figure 3: The specific processes with D-S.

indicates that the trust degree to proposition $A$ is completely determinate when $\operatorname{Bel}(A)=\operatorname{Pl}(A)[11]$.

Reliability function of several independent pieces of evidence exists on the same frame of discernment in the actual application process, and they are not in complete conflict. Then joint reliability function of several pieces of evidence can be obtained by using Dempster evidence fusion rules, and such reliability function is called the direct sum of this evidence reliability function. A composite mass function could be obtained according to D-S fusion rules in consideration for mass function $m_{1}$ and $m_{2}$ of two different pieces of evidence:

$$
\begin{aligned}
& \left(m_{1} \oplus m_{2}\right)(A)=\frac{1}{1-k} \sum_{B \cap C=A} m_{1}(B) m_{2}(C) \\
& \text { where } k=\sum_{B \cap C=\phi} m_{1}(B) m_{2}(C),
\end{aligned}
$$

where $k$ is conflicting belief which represents the level of conflict between the pieces of evidence. If the value of $k$ is large, it illustrates that it has a greater conflict between the evidence. If $k=1$, then it suggests that $m_{1}$ and $m_{2}$ is in total contradiction, and they cannot be combined. The coefficient $1 /(1-k)$ is called normalization factor, which has the function to avoid assigning the probability of nonzero to the empty set $\phi$ in the evidence combination. In this decision-making system, the significance of the weights of conflict is that a big contradiction may exist in vehicle performance attribute, such as the interruption time of powertrain and change rate of vehicle acceleration.

In summary, at first, it needs to calculate, respectively, basic probability assignment function $m(A)$, reliability function $\operatorname{Bel}(A)$, and plausibility function $\operatorname{Pl}(A)$ of various evidence. Then, it is necessary to obtain basic probability assignment function, reliability function, and plausibility function by using evidence composition algorithm under the combined effect of all evidence. Lastly, a decision-making with maximum support degree under the effect of the combined evidence is selected by using certain decision-making rules, and specific processes are shown in Figure 3. Since hybrid powertrain is related to quantitative and qualitative evidence, the authors choose evidential reasoning approach to evaluate the decision-making.
4.2. Calculation Principle of Evidential Reasoning Approach. The evidential reasoning approach put forward by Yang and Sen has a good effect on solving multi-attribute decisionmaking problems which contain both quantitative index and qualitative index; the reliability matrixes based on Figure 3 and evidence combination rule are used to fuse the evaluation value of various index in the problem of multi-attribute decision-making. The model extends the decision-making matrix of the traditional problems, and it makes the traditional decision-making matrix to be divided into part of the quantitative index and part of qualitative index, whose evaluation value of qualitative index is extended from single value to a $N+1$ dimensional vector; every element in the $N+1$ dimensional vector is the experience judgment of confidence level in a linguistic evaluation degree for a evaluation index, that is, $\beta_{n, i}\left(A_{l}\right)[7]$.

Therefore, according to (2), total confidence level of the total attribute of a decision-making $A_{l}$ could be described as follows:

$$
S\left(y\left(A_{l}\right)\right)=\left\{\left(\Theta_{n}, \beta_{n}\left(A_{l}\right)\right), \ldots,\left(\Theta, \beta_{\Theta}\left(A_{l}\right)\right)\right\}
$$

There are both accurate evaluation value of quantitative index and frame of discernment based on different qualitative index which is set to make the decision-making more accurate and more realistic in the practical application under the special driving cycles of hybrid vehicles; the method of inverting the evaluation value of quantitative index into confidence on the total frame of discernment is presented in [12]; the invert rules show as follows:

$$
\begin{aligned}
& \gamma_{n, j}=\frac{H_{j+1}-H_{n, i}}{H_{j+1}-H_{j}}, \quad\left(H_{j} \leq H_{n, i} \leq H_{j+1}\right), \\
& \gamma_{n+1, j}=1-\gamma_{n, j} .
\end{aligned}
$$

where $\gamma_{n, j} \gamma_{n+1, j}$, respectively, are the confidence on $n$ and $n+1$ level for quantitative index.

Concretely speaking, evaluation grade $H_{n, i}$ for quantitative attribute is based on basic attribute (6), and it could be expressed as

$$
H_{n, i}=\left\{\left(H_{l}, \gamma_{l, n}\right), l=1, \ldots, N\right\} .
$$


In addition, there are some uncertainties due to the different quantitative attribute, of driving cycles and vehicle states, for example, there is a significant difference of fuel consumption in different operating conditions:

$$
S\left(e_{i}\right)=\left\{\left(H_{j}, p_{j}\right), j=1,2, \ldots, M_{i}\right\},
$$

where $H_{j}$ refers to the possible value of $e_{i}$ and $p_{j}$ is the probability while the value of $e_{i}$ is $H_{j}, \sum_{j=1}^{M_{i}} p_{j} \leq 1$; combining (13) and (15), we get

$$
\begin{aligned}
& \widetilde{S}\left(e_{i}\right)=\left\{\left(H_{j}, \tilde{\gamma}_{n, i}\right), n=1,2, \ldots, N\right\}, \\
& \tilde{\gamma}_{n, i}=\left\{\begin{array}{c}
\sum p_{j} \gamma_{n, j} \\
\sum p_{j} \gamma_{n, j}+\sum p_{j}\left(1-\gamma_{n, j}\right) \\
\sum p_{j}\left(1-\gamma_{n, j}\right) .
\end{array}\right.
\end{aligned}
$$

In order to simplify the calculation, the quantitative conversion process can be described as

$$
\mathbf{B}_{i}=\mathbf{A}_{i} \times \mathbf{R}_{i} \times \mathbf{p}_{i},
$$

where

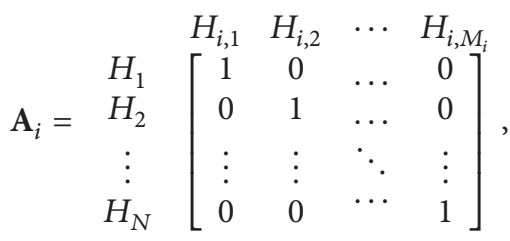

$$
\begin{aligned}
& \mathbf{R}_{i}=\left[\begin{array}{cccc}
\gamma_{1,1} & \gamma_{1,2} & \cdots & \gamma_{1, M_{i}} \\
\gamma_{2,1} & \gamma_{2,2} & \cdots & \gamma_{2, M_{i}} \\
\vdots & \vdots & \ddots & \vdots \\
\gamma_{N, 1} & \gamma_{N, 2} & \cdots & \gamma_{N, M_{i}}
\end{array}\right], \\
& \mathbf{p}_{i}=\left[\begin{array}{c}
p_{1} \\
p_{2} \\
\vdots \\
p_{M_{i}}
\end{array}\right]
\end{aligned}
$$

4.3. Comprehensive Evaluation of Vehicle Performance Based on the Evidential Reasoning Approach. We can make final decision by obtaining the utility value of various propositions or decisions (that is comprehensive evaluation of vehicle performance) from the evidential reasoning approach. Assume that the utility value of evaluation grade $H_{n}$ is indicated by $u\left(H_{n}\right)$. The expected utility $S\left(y\left(A_{l}\right)\right)$ in (12) could be expressed as follows:

$$
u\left(S\left(y\left(A_{i}\right)\right)\right)=\sum_{n=1}^{N} u\left(H_{n}\right) \beta_{n}\left(A_{i}\right)
$$

It shows the evaluation for one decision scheme in $n$th grade of linguistic assessment in (2), but it cannot compare the quality relationship between several schemes through this equation. In order to compare and select schemes, it needs to transform the total confidence for each scheme $A_{l}$ into the utility value as following "deterministic" [13]:

$$
u\left(y\left(A_{i}\right)\right)=\sum_{n=1}^{N} \beta_{n}\left(A_{i}\right) \cdot u\left(H_{n}\right) .
$$

To calculate the utility value of the party $A_{l}$ which is evaluated in the uncertain evaluation, Yang has defined three types of expected utility, which is the maximum utility $u^{+}$, the minimum utility $u^{-}$, and the average expected utility $\bar{u}[14]$ as

$$
u^{+}\left(A_{l}\right)=\sum_{n=1}^{N-1} \beta_{n}\left(A_{l}\right) u\left(H_{n}\right)+\left(\beta_{H}\left(A_{l}\right)+\beta_{N}\left(A_{l}\right)\right) u\left(H_{N}\right)
$$

$$
u^{-}\left(A_{l}\right)=\left(\beta_{1}\left(A_{l}\right)+\beta_{H}\left(A_{l}\right)\right) u\left(H_{1}\right)+\sum_{n=2}^{N} \beta_{n}\left(A_{l}\right) u\left(H_{n}\right),
$$

$$
\bar{u}\left(A_{l}\right)=\frac{u^{+}\left(A_{l}\right)+u^{-}\left(A_{l}\right)}{2} .
$$

Note that if $S\left(e_{i}\right)$ is complete evaluation, then $\beta_{H}\left(A_{l}\right)=0$ and $u\left(S\left(y\left(A_{l}\right)\right)\right)=u^{+}\left(A_{l}\right)=u^{-}\left(A_{l}\right)=\bar{u}\left(A_{l}\right)$. Two decision schemes $A_{l}$ and $A_{k}$ are taken into consideration; just when $u^{-}\left(A_{l}\right)>u^{+}\left(A_{k}\right)$, it could be thought that scheme $A_{l}$ is better than $A_{k}$. When $u^{-}\left(A_{l}\right)=u^{-}\left(A_{k}\right)$ and $u^{+}\left(A_{l}\right)=u^{+}\left(A_{k}\right)$, then it could be concluded that scheme $A_{l}$ is identical with $A_{k}$. This paper ranks decision-making schemes objectively through average utility, scheme $A_{l}$ could be thought better than $A_{k}$ just when $\bar{u}\left(A_{l}\right)>\bar{u}\left(A_{k}\right)$. Of course, in order to obtain reliable decision-making, it needs to improve the completeness of the original data to eliminate errors caused by the uncertainty of data.

\section{An Instance for Decision-Making of Task Conflict}

Table 1 shows that 500 groups of vehicle attribute data are collected under the uphill condition, to calculate the utility value of each group of test data which, respectively, applies different control strategies according to the evidential reasoning approach in Section 4. The following describes specifically the calculation process for evaluating the vehicle utility by using specific experimental data.

Hybrid bus drives in speed of $8 \mathrm{~m} / \mathrm{s}$ with 3 th gear on about $15 \%$ uphill during the test; the engine speed is about $1360(\mathrm{r} / \mathrm{min})$ and the vehicle is running in pure engine mode. According to the vehicle longitudinal dynamics calculation, the maximum acceleration that a vehicle can be provided is about $0.7 \mathrm{~m} / \mathrm{s}^{2}$, which means that the slope can be overcome is $7 \%$. Now we face that the problem involving the choice of task conflict, and the current state in Table 2 contains the above describing vehicle state. To find the best match driving cycle collected in Section 2 by interpolation. The record of performance data relevant to the vehicle which, respectively, applies different decision-making schemes is shown in the column of decision-making scheme in 
TABLE 2: Expected vehicle state parameters relevant to various decision-makings.

\begin{tabular}{|c|c|c|c|c|}
\hline \multirow{2}{*}{ Attribute type } & \multirow{2}{*}{ Definition of attribute } & \multirow{2}{*}{ Current state } & \multicolumn{2}{|c|}{ Decision-making schemes } \\
\hline & & & Multi-energy power split management & Gear changing \\
\hline \multirow{6}{*}{ Quantitative data } & BSFC of ICE & 204 & $\{(202,0.8),(208,0.2)\}$ & $\{(220,0.9)\}$ \\
\hline & Raised of motor temperature & 0 & $\{(10,0.8)\}$ & $\{(0,1)\}$ \\
\hline & Efficiency of motor & 100 & $\{(92,0.9)\}$ & $\{(100,1)\}$ \\
\hline & SOC change of battery $/ \%$ & 0 & $\{(2 \%, 0.7)\}$ & $\{(0,1)\}$ \\
\hline & Gap time of torque & 0 & $\{(0.5,0.9)\}$ & $\{(1.7,0.8)\}$ \\
\hline & Efficiency of battery & 100 & $\{(94,0.7)\}$ & $\{(100,1)\}$ \\
\hline \multirow{3}{*}{ Qualitative estimate } & Average rate of change in acceleration & $\mathrm{W}$ & $\{(G, 0.8),(A, 0.2)\}$ & $\{(E, 0.6),(G, 0.4)\}$ \\
\hline & Average rate of change in throttle & $\mathrm{P}$ & $\{(E, 0.8),(G, 0.2)\}$ & $\{(P, 0.6),(I, 0.4)\}$ \\
\hline & Comfort & A & $\{(E, 0.9),(G, 0.1)\}$ & $\{(P, 0.7),(I, 0.3)\}$ \\
\hline
\end{tabular}

TABLE 3: Expected state parameters after conversion relevant to various decision-making schemes.

\begin{tabular}{lcc}
\hline Definition of attribute & \multicolumn{2}{c}{ Decision-making schemes } \\
BSFC of ICE & Multi-energy power split management & $\{(A, 0.9)\}$ \\
Raised of motor temperature & $\{(G, 0.32),(E, 0.68)\}$ & $\{(T, 1)\}$ \\
Efficiency of motor & $\{(G, 0.8)\}$ & $\{(T, 1)\}$ \\
SOC change of battery/\% & $\{(G, 0.72)\}$ & $\{(T, 1)\}$ \\
Gap time of torque & $\{(G, 0.7)\}$ & $\{(P, 0.26)\}$ \\
Efficiency of battery & $\{(T, 0.9)\}$ & $\{(T, 1)\}$ \\
Average rate of change in acceleration & $\{(G, 0.7)\}$ & $\{(E, 0.6),(G, 0.4)\}$ \\
Average rate of change in throttle & $\{(G, 0.8),(A, 0.2)\}$ & $\{(P, 0.6),(A, 0.4)\}$ \\
Comfort & $\{(E, 0.8),(G, 0.2)\}$ & $\{(P, 0.7),(A, 0.3)\}$ \\
\hline
\end{tabular}

Table 2; the specific form of recording values is \{quantitative or qualitative data, confidence\}.

All quantitative attributes can be expressed equivalently by using the conversion rules, to divide the engine fuel consumption rate into the following several levels according to engine universal performance characteristics map, which, respectively, are corresponding to (6):

$$
\begin{aligned}
H^{1} & =\left\{H_{1,1}, H_{2,1}, H_{3,1}, H_{4,1}, H_{5,1}, H_{6,1}\right\} \\
& =\{270,250,220,210,200,190\} .
\end{aligned}
$$

Similarly, the raised rate of motor temperature, motor efficiency, the change of battery SOC, and the power interruption time and battery efficiency can be equivalently expressed as

$$
\begin{gathered}
H^{2}=\{35,25,15,10,5,0\}, \\
H^{3}=\{80,85,90,94,97,100\}, \\
H^{4}=\{10,6,4,2,1,0\}, \\
H^{5}=\{2,1.8,1.5,1.2,1,0.5\}, \\
H^{6}=\{80,85,90,94,96,100\} .
\end{gathered}
$$

For example, in the multi-energy power split management, the probability of engine BSFC $H_{1}=202$ is $p_{1}=0.8$, and for $H_{1}=208$ is $p_{1}=0.2$, by (13)

$$
\begin{aligned}
& \gamma_{4,1}=\frac{H_{5,1}-H_{1}}{H_{5,1}-H_{4,1}}=\frac{200-202}{200-210}=0.2, \\
& \gamma_{5,1}=1-\gamma_{4,1}=0.8 \\
& \gamma_{4,1}^{\prime}=\frac{H_{5,1}-H_{1}}{H_{5,1}-H_{4,1}}=\frac{200-208}{200-210}=0.8, \\
& \gamma_{5,1}^{\prime}=1-\gamma_{4,1}^{\prime}=0.2 .
\end{aligned}
$$

The final conversion result is

$$
\begin{aligned}
S\left(e_{1}(\mathrm{PSR})\right) & =\left\{\left(H_{4}, 0.48\right),\left(H_{5}, 0.52\right)\right\} \\
& =\{(G, 0.32),(E, 0.68)\} .
\end{aligned}
$$

The expected state parameters relevant to various decision-making are shown in Table 3 after finishing the conversion of quantitative attribute orderly.

Assume that all attributes have the same weight coefficient, which is $w_{i}=1 / 9$ in (4); the evaluation result of these two decision-making under special driving cycles is obtained by using evidential reasoning iteration algorithm and D-S evidence combination rules, as shown in Table 4. 
TABLE 4: Evaluation of whole performance for various decision-making schemes.

Decision-making schemes

Multi-energy power split management

Gear changing

Performance evaluation $\quad\{(W, 0.2),(P, 0.1),(A, 0.4),(G, 0.8),(E, 0.2),(T, 0.3)\} \quad\{(W, 0.3),(P, 0.4),(A, 0.6),(G, 0.4),(E, 0.1),(T, 0.35)\}$

TABle 5: Average utility and rank of the two decision-making schemes.

\begin{tabular}{lcc}
\hline & \multicolumn{2}{c}{ Decision-making scheme } \\
& $\begin{array}{c}\text { Gear } \\
\text { changing }\end{array}$ & $\begin{array}{c}\text { Multi-energy power split } \\
\text { management }\end{array}$ \\
\hline Rank of utility & 0.8851 & 0.6615 \\
& 1 & 2 \\
\hline
\end{tabular}

Evaluating the utility related to various decision-making schemes by using the recursive method based on the evaluation results of whole performance. Firstly, it needs the normalization process for the utilization of various evaluation grades, assuming that each grade has the same position of the utility. We could determine the utility of the two decisionmaking schemes by (19) and (23) (if the attribute information is complete in Table 2 , then the maximum utility, minimum utility, and average utility of various decision-making are equal). To provide a direct basis for the solution of the two-task conflict, the rank of vehicular performance caused by the two decision-making can be determined based on utility value, as shown in Table 5 . It can determine that gear changing is the optimal decision-making under this diving cycle for overcoming the load, which can make vehicular performance to be the optimal.

According to the above evaluation of calculating method, the best decision for 500 groups' typical experimental data under ramp condition is obtained via making corresponding software to select the driving cycle which is closest to the current vehicle state from 500 kinds of typical driving cycles by the method of interpolating selecting when the specific application and then to make decision according to the evaluation results. The results of Chongqing public transportation line 818 tests confirm that the proposed decision strategy is correct and effective, and fuel economy improvement has been achieved compared with applying the decision of gear changing or multi-energy power split management all the time for hybrid electric bus.

\section{Conclusions}

(1) One of the issues is associated with conflict decision between shift schedule and power management. This issue is addressed in this study for parallel hybrid electric vehicle (PHEV) under special driving cycle.

(2) Using the evidential reasoning approach based on Dempster-Shafer evidence combination theory, the evaluation system of whole performance for hybrid system under typical special driving cycles is established. The optimal decision table used for real-time applications is obtained.
(3) By calculating the instance, the interruption duration of hybrid power AMT shifting process plays a crucial role in the conflict decision-making. It is also necessary to put an in-depth and meticulous research on shifting process control. In order to reach at a point for reducing the time of shifting process significantly and improving vehicular comprehensive performance in the decision-making time of downshift under special driving cycles, a new technology for shift process control is proposed $[15,16]$.

\section{Acknowledgment}

This work was supported by the National High Technology Research and Development Program of China (Grant no. 2011AA11A252).

\section{References}

[1] W. Rui-lin and S. Jian-wu, Urban Road Design, China Communications Press, 2003.

[2] L. Qiao, M. Sato, K. Abe, and H. Takeda, "Self-supervised learning algorithm of environment recognition in driving vehicle," IEEE Transactions on Systems, Man, and Cybernetics A, vol. 26, no. 6, pp. 843-850, 1996.

[3] S.-I. Jeon, S.-T. Jo, Y.-I. Park, and J.-M. Lee, "Multi-mode driving control of a parallel hybrid electric vehicle using driving pattern recognition," Journal of Dynamic Systems, Measurement and Control, vol. 124, no. 1, pp. 141-149, 2002.

[4] A. D. Abdollahi, "An intelligent control strategy in a parallel hybrid vehicle," in Proceedings of the IEEE Conference on Electric and Hybrid Vehicles (ICEHV'06), pp. 1-2, December 2006.

[5] M. Montazeri-Gh, A. Ahmadi, and M. Asadi, "Driving condition recognition for genetic-fuzzy HEV control," in Proceedings of the 3rd International Workshop on Genetic and Evolving Fuzzy Systems (GEFS '08), pp. 65-70, March 2008.

[6] V.-N. Huynh and Y. Nakamori, "A satisfactory-oriented approach to multiexpert decision-making with linguistic assessments," IEEE Transactions on Systems, Man, and Cybernetics B, vol. 35, no. 2, pp. 184-196, 2005.

[7] J.-B. Yang and P. Sen, "General multi-level evaluation process for hybrid MADM with uncertainty," IEEE Transactions on Systems, Man and Cybernetics, vol. 24, no. 10, pp. 1458-1473, 2005.

[8] J.-B. Yang and M. G. Singh, "Evidential reasoning approach for multiple-attribute decision making with uncertainty," IEEE Transactions on Systems, Man and Cybernetics, vol. 24, no. 1, pp. 1-18, 1994.

[9] Z. Mi, Research on Some Problems in the Multiple Attribute Decision Making Based on Evidential Reasoning Approach, Hefei University of Technology, 2009.

[10] G. Shafer, A Mathematical Theory of Evidence, Princeton University Press, Princeton, NJ, USA, 1976. 
[11] H. Jing-feng, The Assessment Model and Implementation for Quality Performance, Northwestern Polytechnical University, 2006.

[12] D. Thierry, A k-Nearest Neighbor Classification Rule Based on Dempster-Shafer Theory, Springer, New York, NY, USA, 2008.

[13] R. R. Yager, "On the dempster-shafer framework and new combination rules," Information Sciences, vol. 41, no. 2, pp. 93-137, 1987.

[14] J.-B. Yang and D.-L. Xu, "On the evidential reasoning algorithm for multiple attribute decision analysis under uncertainty," IEEE Transactions on Systems, Man, and Cybernetics A, vol. 32, no. 3, pp. 289-304, 2002.

[15] H. Zhang, J. Wang, and Y. Shi, "Robust $H_{\infty}$ sliding-mode control for Markovian jump systems subject to intermittent observations and partially known transition probabilities," Systems \& Control Letters, vol. 62, no. 12, pp. 1114-1124, 2013.

[16] H. Zhang, Y. Shi, and J. Wang, "Observer-based tracking controller design for networked predictive control systems with uncertain Markov delays," International Journal of Control, vol. 86, no. 10, pp. 1824-1836, 2013. 


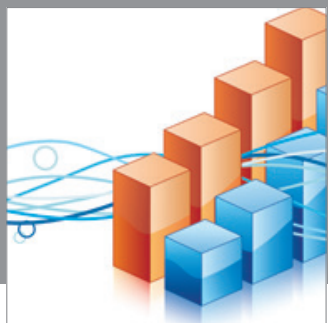

Advances in

Operations Research

mansans

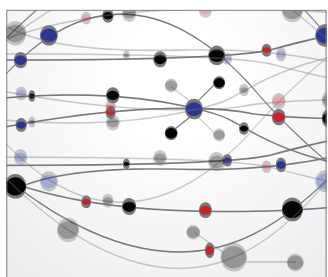

The Scientific World Journal
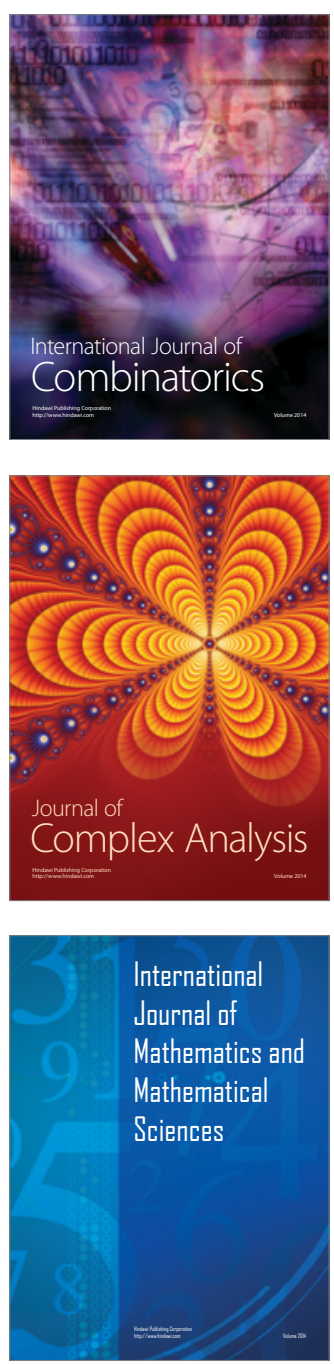
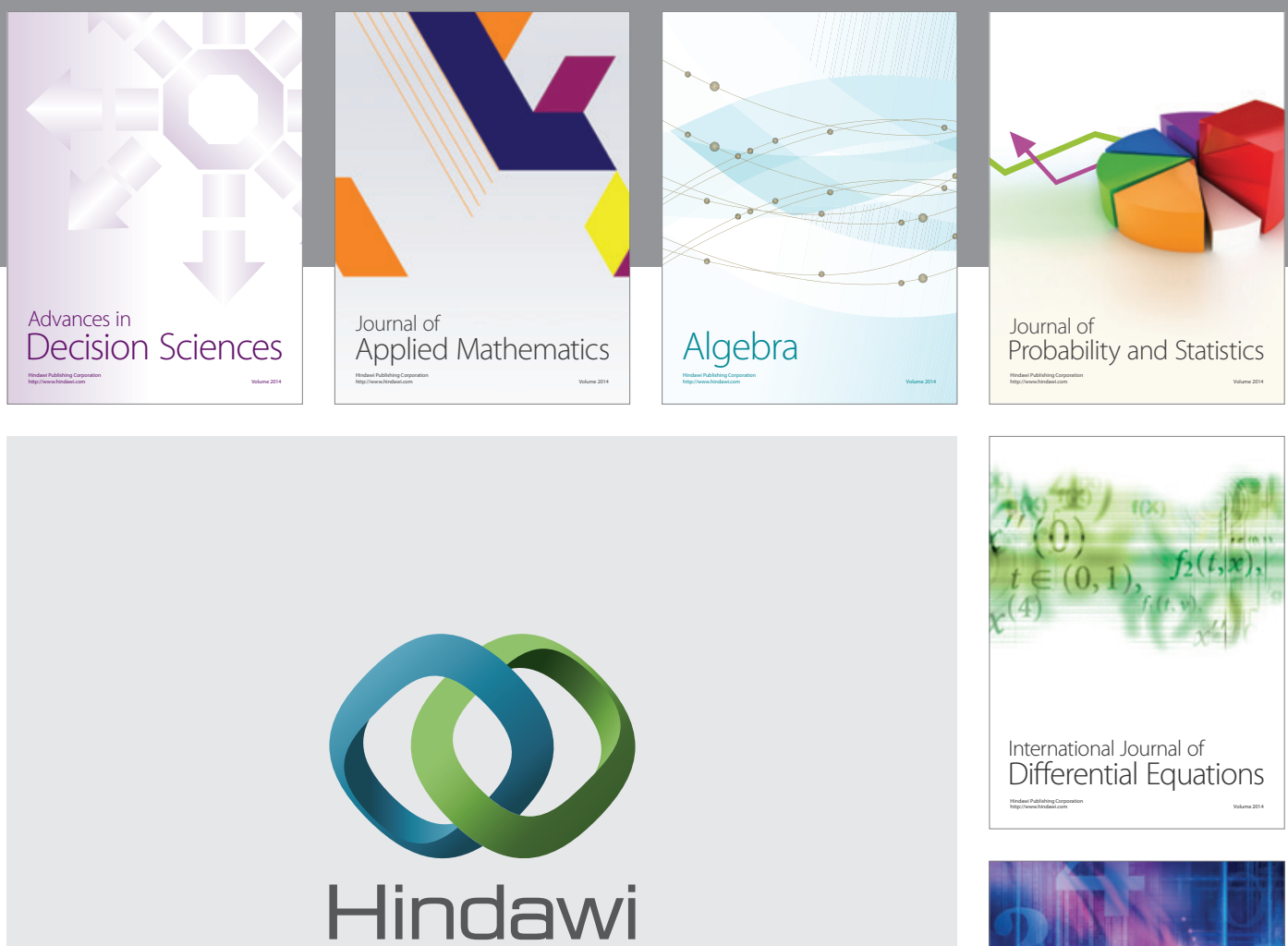

Submit your manuscripts at http://www.hindawi.com
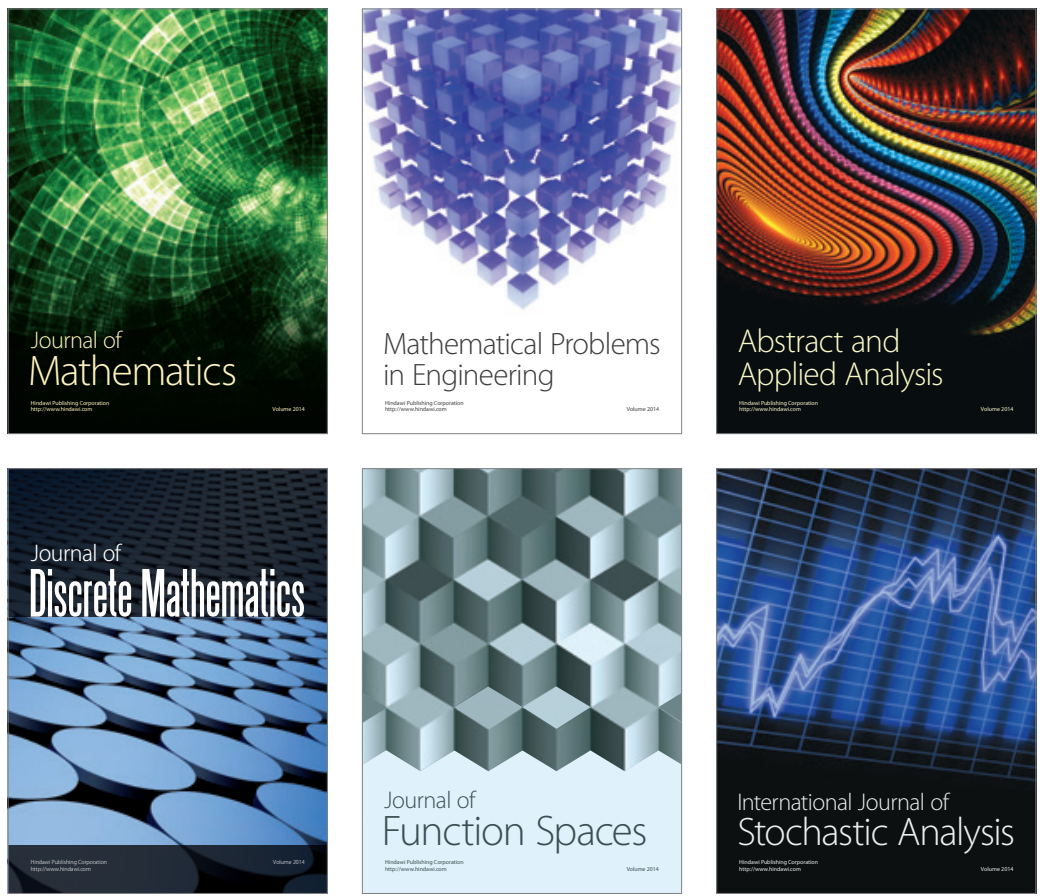

Journal of

Function Spaces

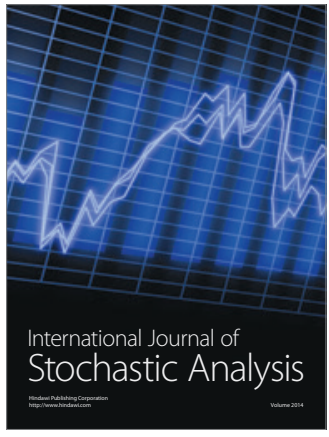

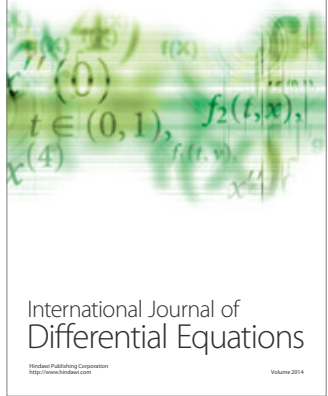
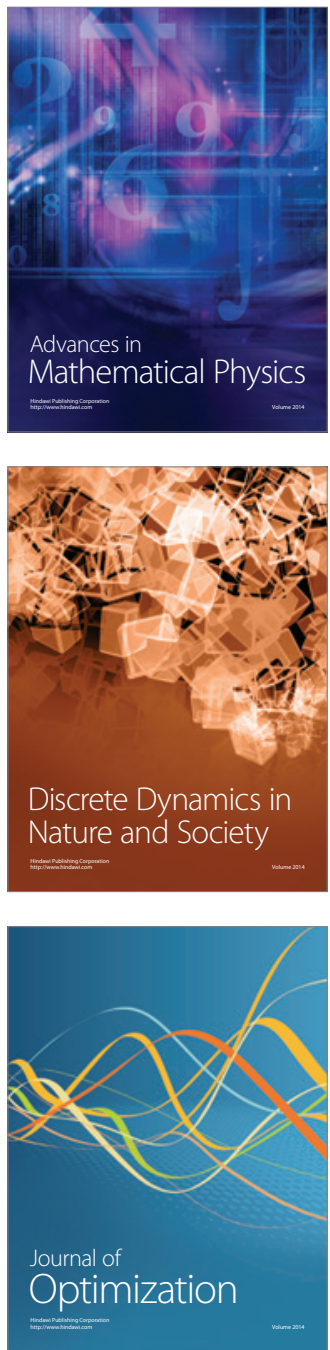